\title{
SIMULACIÓN DE MONTE CARLO APLICADA A LA ESTIMACIÓN DE DEPRESIONES RÁPIDAS DE LA TENSIÓN EN REDES ELÉCTRICAS
}

\section{MONTE CARLO SIMULATION APPLIED TO THE ESTIMATION OF VOLTAGE DIPS IN ELECTRIC NETWORKS}

\author{
Miguel Arias Albornoz ${ }^{1} \quad$ Marcelo Aedo Ruz Gabriel Olguín Parada \\ Recibido 23 de marzo de 2006, aceptado 14 de enero de 2008 \\ Received: March 23, 2006 Accepted: January 14, 2008
}

\begin{abstract}
RESUMEN
En este trabajo se aplica el método de simulación de Monte Carlo (MC) para estimar el número de depresiones rápidas de tensión (dips) esperadas en barras de una red eléctrica. Las estimaciones obtenidas a través de MC se comparan con los resultados de otro método de cálculo conocido como Método de Posiciones de Falla (MPF). Entre los resultados se muestra tanto la convergencia del algoritmo MC a los valores de largo plazo del método MPF como la distribución completa de frecuencias para diferentes eventos, lo cual representa información valiosa para apoyar la toma de decisiones sobre el empleo de equipos sensibles a este tipo de perturbación.
\end{abstract}

Palabras clave: Simulación de Monte Carlo, calidad de energía, estimación estocástica, dips de voltaje.

\begin{abstract}
In this work, the Monte Carlo simulation method $(M C)$ is applied to estimate the number of expected voltage dips in the nodes of an electric network. The estimations obtained through MC are compared with the results of another method of calculation, known as Failure Position Method (MPF). In the results, both the convergence of the algorithm with the longterm values of the MPF method and the complete distribution of frequencies for different events are shown. This represents valuable information to support the decision-making process for equipment that is sensitive to this type of perturbation.
\end{abstract}

Keywords: Monte Carlo simulation, power quality, stochastic estimation, voltage dips.

\section{INTRODUCCIÓN}

Las depresiones rápidas del valor rms de la tensión (dips de voltaje) son originadas por circulación de altas corrientes en la red, que afectan los voltajes en tiempos que van desde unos ciclos a algunos segundos. Constituyen una de las perturbaciones más severas en calidad de energía ya que pueden provocar la detención completa de una planta industrial [1]. Entre las causas que provocan circulación de altas corrientes se pueden señalar la energización de motores de gran potencia, energización de transformadores y cortocircuitos. Los dips más severos son provocados por cortocircuitos, especialmente en niveles de transmisión.

En la literatura técnica internacional se encuentran diversos trabajos que estudian el fenómeno de dips, pero en Chile el tema es poco conocido. Trabajos que reportan problemas específicos de dips, analizando el efecto de las depresiones de tensión sobre consumos industriales [2], concluyen que la mayoría de los eventos que afectan los procesos productivos son ocasionados por fallas en la red de suministro. En [2] se analiza el efecto de las depresiones de tensión sobre cargas particulares, como accionamientos de velocidad controlada, controladores lógicos programables y contactores de motores. En [3] se analiza la sensibilidad de los procesos industriales, correlacionando las paradas de los procesos con diversas maneras de caracterizar las depresiones de tensión.

El efecto de dips sobre cargas sensibles hace necesario tratarlas como un problema de compatibilidad electromagnética entre la carga y la fuente de alimentación, lo que se puede realizar, por ejemplo, a través de la curva CBEMA [5]. Por este motivo, es necesario estimar el número de dips que

\footnotetext{
1 Departamento de Ingeniería Eléctrica. Universidad de Santiago de Chile. Av. Ecuador 3519. Casilla 10233. Santiago, Chile. E-mail: miguel.arias@usach.cl
} 
se puede presentar en una barra de interés, clasificandolos generalmente según magnitud y duración.

Bollen, en [1], realiza un estudio detallado de la incidencia de las depresiones de tensión sobre diversos consumos que utilizan electrónica de potencia, y también describe diversos métodos para caracterizar y evaluar los disturbios. Señala que una forma de registrar la cantidad de dips que ocurren en la red es a través del monitoreo; sin embargo, para obtener buenos niveles de precisión se requiere un tiempo elevado de registro (tabla 1), que se incrementa en la medida que disminuye la tasa promedio de eventos, constituyendo un proceso costoso que demanda mucho tiempo.

Tabla 1. Tiempos de registro para monitoreo de dips.

\begin{tabular}{|l|c|c|c|}
\cline { 2 - 4 } \multicolumn{1}{c|}{} & \multicolumn{3}{c|}{ \% de error } \\
\hline Tasa promedio & $50 \%$ & $10 \%$ & $2 \%$ \\
\hline 1 evento/día & 2 semanas & 1 año & 25 años \\
\hline 1 evento/semana & 4 meses & 7 años & 200 años \\
\hline 1 evento/mes & 1 año & 30 años & 800 años \\
\hline 1 evento/año & 16 años & 400 años & 10000 años \\
\hline
\end{tabular}

Debido a lo elevado de estos requerimientos, es necesario aplicar métodos de predicción, uno de los cuales es la estimación estocástica [6]. Por esta razón, en el presente trabajo se aplica el método de simulación Monte Carlo, que combina el cálculo determinístico de los voltajes residuales vistos en una barra del sistema, debido a una falla en algún punto de la red, con la probabilidad de que la falla se presente en dicho punto [7]. La ventaja de Monte Carlo es que, además de los índices correspondientes a los valores esperados de largo plazo, también entrega la distribución completa de frecuencias para un índice específico (dip de una magnitud dada). Otro enfoque para determinar el número de eventos esperados de cierta magnitud en algunas barras del sistema lo constituye el método de posiciones de falla [8]; sin embargo, este método no entrega la variabilidad de los índices.

Para realizar la estimación del número de eventos, en este trabajo solo se considera la magnitud de dips que superan un valor dado, mostrando los resultados en gráficos de frecuencia acumulada.

\section{MODELACIÓN DEL SISTEMA}

Para determinar los voltajes residuales en una barra, cuando ocurren cortocircuitos en el sistema, se puede utilizar la matriz impedancia de barras y el teorema de Thevenin generalizado [9]. De esta forma, para un cortocircuito en una barra $p$ del sistema, se tiene:

$$
\left[\begin{array}{c}
V_{1} \\
\vdots \\
V_{p} \\
\vdots \\
V_{p}
\end{array}\right]=\left[\begin{array}{ccccc}
Z_{11} & \cdots & Z_{1 p} & \cdots & Z_{1 n} \\
\vdots & & \vdots & & \vdots \\
Z_{p 1} & \cdots & Z_{p p} & \cdots & Z_{p n} \\
\vdots & & \vdots & & \vdots \\
Z_{n 1} & \cdots & Z_{n p} & \cdots & Z_{n n}
\end{array}\right] \cdot\left[\begin{array}{c}
0 \\
\vdots \\
I_{p} \\
\vdots \\
0
\end{array}\right]
$$

Donde,

$\mathrm{Z}_{k p}$ : Impedancia de transferencia entre cualquier par de barras $k$ y $p$ del sistema.

$\mathrm{Z}_{p p}$ :Impedancia equivalente vista desde la barra $p$.

Por ejemplo, el voltaje en barra $k$ durante una falla en la barra $p$, cuyo valor está relacionado con la impedancia de transferencia entre estas dos barras, es:

$$
V_{k}=Z_{k p} \cdot I_{p}
$$

La impedancia de transferencia entre la barra $k$ y la barra $p$, es:

$$
Z_{k p}=\frac{V_{k}}{I_{p}}
$$

Como la matriz de impedancia de barras es llena, una falla en cualquier punto de la red afectará el voltaje en todas las barras del sistema. En particular, el efecto de una falla balanceada en barra $p$ sobre el voltaje de una barra $m$ cualquiera, considerando la impedancia de falla $Z_{f}=0$ (peor condición), se determina a partir de:

$$
\left[\begin{array}{c}
V_{1} \\
\vdots \\
V_{p} \\
\vdots \\
V_{p}
\end{array}\right]=\left[\begin{array}{c}
E_{1} \\
\vdots \\
E_{p} \\
\vdots \\
E_{n}
\end{array}\right]-\left[\begin{array}{ccccc}
Z_{11} & \cdots & Z_{1 p} & \cdots & Z_{1 n} \\
\vdots & & \vdots & & \vdots \\
Z_{p 1} & \cdots & Z_{p p} & \cdots & Z_{p n} \\
\vdots & & \vdots & & \vdots \\
Z_{n 1} & \cdots & Z_{n p} & \cdots & Z_{n n}
\end{array}\right] \cdot\left[\begin{array}{c}
0 \\
\vdots \\
I_{p} \\
\vdots \\
0
\end{array}\right]
$$

Considerando ahora que los voltajes prefalla son todos igual a $1<0^{\circ} \mathrm{pu}$ (sistema en vacío, sin cargas), se obtiene:

$$
V_{m p}=1-\frac{Z_{m p}}{Z_{p p}}
$$

Donde:

$V_{m p}=$ Voltaje en barra $m$ durante falla trifásica en barra $p$. $\mathrm{Z}_{m p}^{m p}$ : Impedancia entre barra $m$ y punto de falla $p$. 
Para fallas desbalanceadas se utilizan las redes de secuencia, interconectadas según el tipo de falla: fase a tierra (L), fase-fase (L-L) o fase-fase a tierra (L-L-g). Expresando la ecuación (4) en componentes de secuencia, se tiene:

$$
\left[\begin{array}{c}
V_{k f}^{0} \\
V_{k f}^{1} \\
V_{k f}^{2}
\end{array}\right]=\left[\begin{array}{c}
0 \\
V_{p r e f(k)}^{1} \\
0
\end{array}\right]-\left[\begin{array}{ccc}
Z_{k f}^{0} & 0 & 0 \\
0 & Z_{k f}^{1} & 0 \\
0 & 0 & Z_{k f}^{2}
\end{array}\right] \cdot\left[\begin{array}{c}
I_{f}^{0} \\
I_{f}^{1} \\
I_{f}^{2}
\end{array}\right]
$$

Para cada tipo de falla, los voltajes en componentes de secuencia se transforman a componentes de fase. Así, para una falla fase a tierra se obtiene:

$$
\begin{aligned}
& V_{k f}^{a}=V_{a}^{p r e f(k)}-\left(Z_{k f}^{0}+Z_{k f}^{1}+Z_{k f}^{2}\right) \frac{V_{a}^{p r e f(f)}}{Z_{f f}^{0}+Z_{f f}^{1}+Z_{f f}^{2}} \\
& V_{k f}^{b}=a^{2} V_{a}^{\operatorname{pref}(k)}-\left(Z_{k f}^{0}+a^{2} Z_{k f}^{1}+a Z_{k f}^{2}\right) \frac{V_{a}^{\operatorname{pref}(f)}}{Z_{f f}^{0}+Z_{f f}^{1}+Z_{f f}^{2}} \\
& V_{k f}^{c}=a V_{a}^{\text {pref }(k)}-\left(Z_{k f}^{0}+a Z_{k f}^{1}+a^{2} Z_{k f}^{2}\right) \frac{V_{a}^{p r e f(f)}}{Z_{f f}^{0}+Z_{f f}^{1}+Z_{f f}^{2}}
\end{aligned}
$$

Para fallas fase-fase:

$$
\begin{gathered}
V_{k f}^{a}=V_{a}^{p r e f(k)} \\
V_{k f}^{b}=a^{2} V_{a}^{p r e f(k)}+j \sqrt{3} \cdot Z_{k f}^{1} \cdot \frac{V_{a}^{\operatorname{pref}(f)}}{Z_{f f}^{1}+Z_{f f}^{2}} \\
V_{k f}^{c}=a V_{a}^{p r e f(k)}-j \sqrt{3} \cdot Z_{k f}^{1} \cdot \frac{V_{a}^{p r e f(f)}}{Z_{f f}^{1}+Z_{f f}^{2}}
\end{gathered}
$$

Para fallas fase-fase a tierra:

$$
\begin{aligned}
& V_{k f}^{a}=V_{a}^{p r e f(k)}+\frac{V_{a}^{\text {pref }(f)}\left[\left(Z_{k f}^{0}-Z_{k f}^{1}\right) Z_{f f}^{0}+\left(Z_{k f}^{0}-Z_{k f}^{1}\right) Z_{f f}^{2}\right.}{Z_{f f}^{1} Z_{f f}^{0}+Z_{f f}^{2} Z_{f f}^{1}+Z_{f f}^{0} Z_{f f}^{2}} \\
& V_{k f}^{b}=a^{2} V_{a}^{p r e f(k)}+\frac{V_{a}^{\text {pref }(f)}\left[\left(a Z_{k f}^{0}-a^{2} Z_{k f}^{1}\right) Z_{f f}^{0}+\left(Z_{k f}^{0}-a^{2} Z_{k f}^{1}\right) Z_{f f}^{2}\right.}{Z_{f f}^{1} Z_{f f}^{0}+Z_{f f}^{2} Z_{f f}^{1}+Z_{f f}^{0} Z_{f f}^{2}} \\
& V_{k f}^{c}=a V_{a}^{p r e f(k)}+\frac{V_{a}^{p r e f(f)}\left[\left(a^{2} Z_{k f}^{0}-a Z_{k f}^{1}\right) Z_{f f}^{0}+\left(Z_{k f}^{0}-a Z_{k f}^{1}\right) Z_{f f}^{2}\right.}{Z_{f f}^{1} Z_{f f}^{0}+Z_{f f}^{2} Z_{f f}^{1}+Z_{f f}^{0} Z_{f f}^{2}}
\end{aligned}
$$

\section{Efecto de la conexión de transformadores}

Para la caracterización de dips interesa que las ecuaciones reflejen los voltajes trifásicos durante la falla con respecto a los voltajes trifásicos prefalla en el punto de observación [10], frente a cualquier condición. Para ello es necesario corregir las ecuaciones anteriores, considerando la presencia de transformadores en conexión estrella-delta.

Como se sabe, una falla fase-tierra ocurrida en el lado estrella de un transformador delta-estrella se refleja como una falla fase-fase en el lado en delta. También se sabe que en transformadores conectados en delta-estrella o delta-estrella aterrizada la secuencia cero no pasa de un lado al otro del transformador; el voltaje de secuencia positiva es rotado $30^{\circ}$ en una dirección, mientras que el voltaje de secuencia negativa es rotado en la misma cantidad, pero en sentido contrario [10].

Para transformadores construidos de acuerdo a la norma ANSI/IEEE [11], ya sea en conexión delta-estrella o estrella-delta, el voltaje fase-neutro de secuencia positiva en el lado de baja tensión retrasa $30^{\circ}$ al voltaje fase-neutro del lado de alta tensión; es decir, según esta norma, las conexiones posibles son $\mathrm{Dy}_{1}$ o $\mathrm{Yd}_{1}$. Cuando se trata de la secuencia negativa, por tener un sentido de giro opuesto a la secuencia positiva, el voltaje que pasa del lado de alta tensión al lado de baja tensión adelanta $30^{\circ}$ con respecto a la secuencia positiva. En resumen, el fasor de voltaje fase-neutro de secuencia positiva gira $30^{\circ}$ en un sentido y el fasor de voltaje fase-neutro de secuencia negativa gira $30^{\circ}$ en el sentido contrario, respecto a la secuencia positiva del lado contrario del transformador, como se ilustra en figura 1.

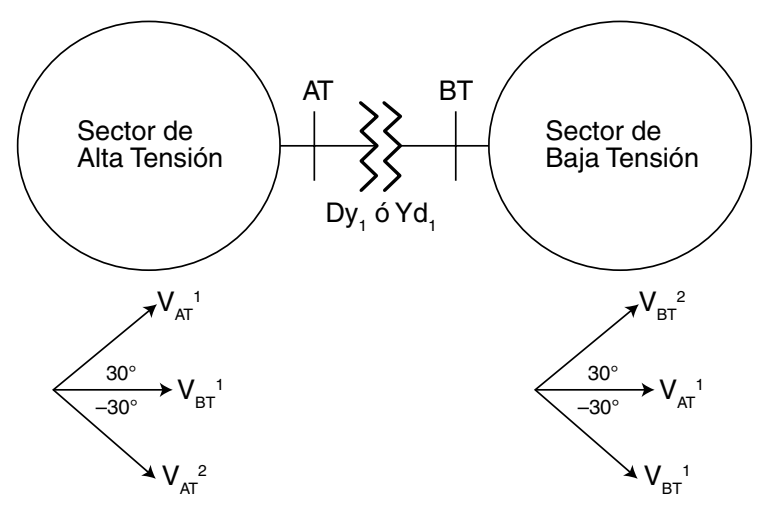

Figura 1. Diagrama fasorial de voltajes de secuencia positiva y negativa para un transformador delta-estrella. 
Para una falla desbalanceada ocurrida en el lado de AT y observada en el lado de BT (esquema fasorial de la derecha en figura 1), el voltaje de secuencia negativa está a $+60^{\circ}$ de la referencia, que en este caso es el voltaje de secuencia positiva prefalla del lado de baja tensión. Para una falla desbalanceada ocurrida en el lado de baja tensión y vista en el lado de alta tensión (esquema fasorial de la izquierda en figura 1), el voltaje de secuencia negativa rota en $-60^{\circ}$ con respecto a la referencia de la barra de observación (ahora la referencia es el voltaje prefalla de secuencia positiva del lado de alta tensión). Esta rotación de $\pm 60^{\circ}$ en la componente de secuencia negativa, con respecto a la secuencia positiva prefalla, puede ser incluida en las ecuaciones usando el operador de Fortescue $a$, de la siguiente manera:

$$
\begin{aligned}
-a^{2} & =1 \angle 60^{\circ} \\
-a & =1 \angle-60^{\circ}
\end{aligned}
$$

\section{Efecto de la impedancia del tramo de línea en falla}

Para considerar la impedancia del tramo de línea en falla, se pueden utilizar ecuaciones que modifican las impedancias de transferencia y de Thevenin vistas desde el punto de falla. Estas ecuaciones, que evitan tener que ampliar la matriz impedancia de barras [12], son las siguientes:

$$
\begin{aligned}
& Z_{m p}=\left(Z_{m j}-Z_{m k}\right) \lambda+Z_{m k} \\
& Z_{p p}=\left(Z_{k k}+Z_{j j}-2 \cdot Z_{k j}-z_{k j}\right) \lambda^{2}+\left(2 \cdot Z_{k j}-2 \cdot Z_{k k}+z_{k j}\right) \lambda+Z_{k k}
\end{aligned}
$$

\section{Donde:}

$$
\begin{gathered}
\lambda=\frac{d_{k p}}{d_{k j}} \quad \begin{array}{c}
\text { Razón entre el tramo desde la barra de origen } k \mathrm{al} \\
\text { punto de falla } p \text { y el largo total de la línea. }
\end{array} \\
\mathrm{z}_{k j} \quad: \text { Impedancia de la línea entre barras } \mathrm{k}-\mathrm{j} .
\end{gathered}
$$

\section{MÉTODO DE POSICIONES DE FALLA}

La aplicación del método de posiciones de falla considera cortocircuitos balanceados y desbalanceados, en un número determinado de posiciones a lo largo de las líneas y en las barras del sistema. Para cada falla se calcula y almacena el voltaje en una barra de interés. La frecuencia de ocurrencia de fallas en alguna posición específica se obtiene a través de la tasa de falla de líneas y de barras [13].
Para el conteo de dips en una barra mediante este método es necesario conocer la magnitud del voltaje en dicha barra debido a fallas ocurridas en todas las posiciones de falla del sistema. Se obtiene así un vector para cada barra, de largo igual al número de posiciones de falla. Ordenando los vectores de todas las barras, para fallas balanceadas, se obtiene una matriz [Vdip], mientras que para fallas desbalanceadas se obtienen tres matrices: [Vdipa], [Vdipb] y [Vdipc]. Puesto que a un cliente le interesa solo el menor voltaje que se puede presentar en la barra donde se encuentran conectados sus equipos, la magnitud del dip a considerar será el menor entre las tres fases en la barra de interés.

\section{SIMULACIÓN DE MONTE CARLO}

Se puede considerar como un método general, que resuelve un problema matemático a través del estudio estadístico de resultados entregados por repetición de un experimento. La simulación de Monte Carlo no resuelve las ecuaciones que describen un modelo, solo se simula y se observa el comportamiento estocástico de estos [1]. Por lo tanto, se requiere considerar un periodo de tiempo suficientemente largo que asegure la convergencia de los resultados (aquí se han usado 1.000 años). Cada estudio utilizando simulación Monte Carlo requiere de la generación de números aleatorios apropiados, sorteados de una distribución de probabilidad dada [6]. Con los resultados obtenidos de la simulación se determinan los valores esperados y la variabilidad de los índices.

Para realizar la simulación se debe modelar el comportamiento de las variables aleatorias que están involucradas, en este caso: el tiempo medio para falla, la posición de la falla y el tipo de falla. El tiempo medio para falla (TMF) se define como el inverso de la tasa de falla, es decir, el tiempo promedio esperado para que ocurra una falla en algún elemento del sistema; en este caso particular, en las líneas y barras de una red eléctrica. Como se debe considerar un periodo de tiempo suficientemente largo, el TMF se ajusta a una distribución normal de probabilidad típica, con media igual al inverso de la tasa de falla (por definición) y una desviación estándar de $30 \%$. La posición de falla se considera distribuida uniforme sobre la línea, de forma que cualquier punto sobre ella, incluyendo las barras, tiene la misma posibilidad de presentar una falla.

El algoritmo usado para la estimación es el siguiente:

1. Definir una barra de observación (barra m).

2. Definir tiempo de simulación (se han usado 1.000 años para obtener porcentaje de error pequeño, según tabla 1). 
3. Para una línea, generar un TMF desde la distribución de probabilidad.

4. Calcular el tiempo acumulado hasta el momento.

5. Para cada posición, definir el lugar sobre la línea donde se simulará la falla.

6. Para cada falla definir el tipo de falla: balanceada, L, L-L, L-L-g.

7. Calcular el voltaje residual en la barra $\mathrm{m}$.

8. Generar TMF y sumarlo al calcuado en 4. Si el tiempo acumulado es menor que el definido en 2, ir a 5. En caso contrario, continúe.

9. Repetir este algoritmo para todas las líneas del sistema.

10. Realizar estadísticas y obtener resultados.

\section{SIMULACIONES Y RESULTADOS}

\section{Sistema ejemplo}

Para realizar los estudios de simulación se ha utilizado el sistema IEEE-24 (Reability Test System), que se muestra en la figura 2, el cual tiene 24 barras y 33 líneas en 230 $\mathrm{kV}$ y $138 \mathrm{kV}$. Los datos de este sistema se encuentran en referencia [8]. La distribución de fallas consideradas en este trabajo son: balanceadas: $5 \%$, L: $80 \%$, L-L: $5 \%$, y L-L-g: $10 \%$.

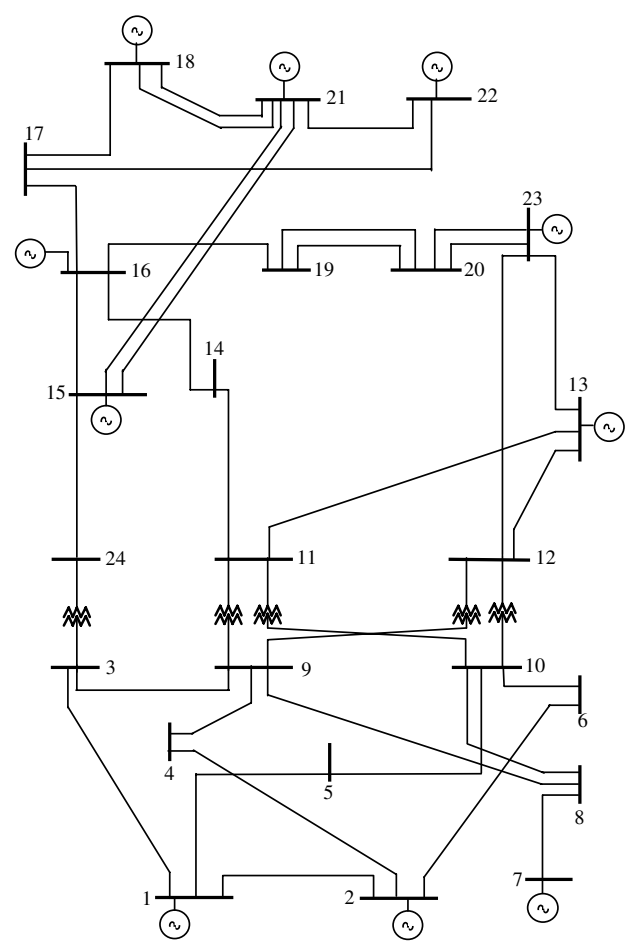

Figura 2. Diagrama unilineal sistema IEEE-24 barras.

\section{Resultados con método de posiciones de falla}

Se estudian dos casos. El caso A con 24 posiciones de falla y el caso B con 231 posiciones de falla (siete posiciones por línea).

Como en la referencia [8] se indica la tasa de falla total para cada línea del sistema, es necesario hacer una estimación de una tasa de falla para cada posición de falla a considerar. Para el caso A, esto se logra asignando la mitad de la tasa de falla de cada línea a cada barra entre las que se encuentra conectada dicha línea. La tasa de falla resultante en una barra será la sumatoria de los aportes de cada línea conectada a ella. Para el caso B, la tasa de falla de cada línea se divide por siete, y se asume que cada posición de falla a lo largo de la línea tiene la misma tasa.

En la figura 3 se resumen las estimaciones de las frecuencias acumuladas obtenidas para el Caso A. El gráfico de frecuencias representa el número de eventos esperados por año o SARFI-X (System Average RMS Variation Frequency Index up to $X$ ) para las magnitudes mostradas en el eje $x$. Se observa que la barra que tiene mejor desempeño es la barra 7, y la que tiene peor desempeño es la barra 4, ya que presenta mayor número de eventos severos, menores de $0.7 \mathrm{pu}$.

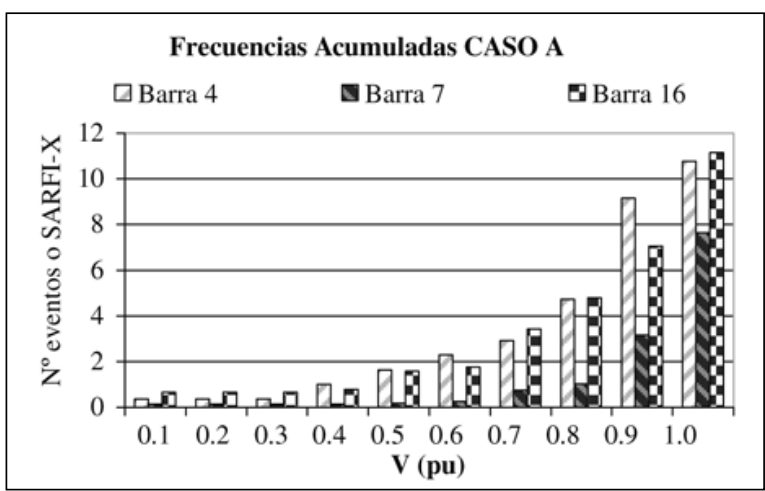

Figura 3. Frecuencias acumuladas caso A en barras 4; 7 y 16 del sistema de prueba.

Los resultados del caso $\mathrm{B}$ se resumen en las figuras siguientes. Los valores de la figura 4, para magnitudes menores o iguales a $0.95 \mathrm{pu}$, son menores que los de la figura 3, debido a que la primera es una estimación más gruesa por considerar solo 24 posiciones de falla. 


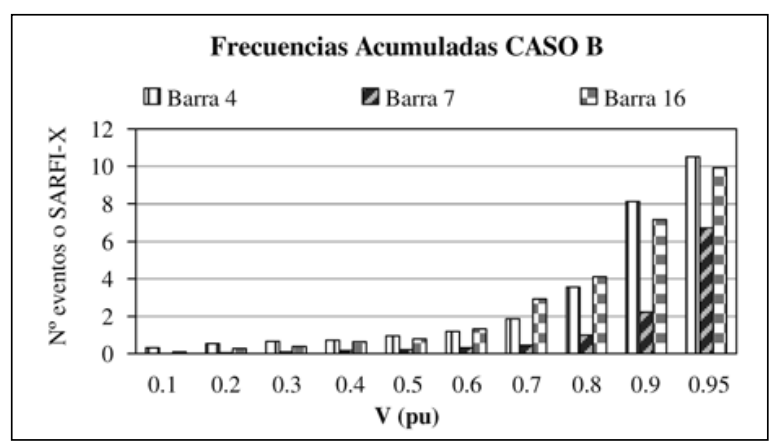

Figura 4. Frecuencias acumuladas caso $\mathrm{B}$ en barras 4, 7 y 16 del sistema de prueba.

Una estadística del sistema se resume en la tabla 2, donde se puede observar que el número de eventos esperados en el sistema completo, de magnitud menor o igual a 0.7 pu es 1.91. Es decir, la suma de los eventos menores o iguales a $0.7 \mathrm{pu}$, obtenidos en cada barra, dividido por el número de barras del sistema es 1.91 eventos.

Tabla 2. Estadística del sistema para los casos A y B.

\begin{tabular}{|c|c|c|c|c|c|c|c|}
\hline & \multicolumn{6}{|c|}{ Estadísticas del Sistema por Magnitud } \\
\hline & & 0.1 & 0.3 & 0.5 & 0.7 & 0.9 & 0.95 \\
\hline \multirow{2}{*}{$\underset{A}{C A S O}$} & Promedio & 0.51 & 0.71 & 1.23 & 2.48 & 7.45 & 10.16 \\
\hline & Perc-95\% & 0.79 & 1.06 & 1.99 & 3.63 & 9.40 & 11.17 \\
\hline \multirow{2}{*}{$\begin{array}{c}\text { CASO } \\
\boldsymbol{B}\end{array}$} & Promedio & 0.14 & 0.43 & 0.88 & 1.91 & 6.75 & 9.84 \\
\hline & Perc-95\% & 0.33 & 0.66 & 1.64 & 2.93 & 8.80 & 11.57 \\
\hline
\end{tabular}

El percentil-95 representa el valor que no es superado por el $95 \%$ de todos los números de una muestra. Para los dips menores o iguales a 0.9 pu el percentil-95 es 8.8 eventos por año, es decir, de las estimaciones realizadas en las 24 barras, el $95 \%$ de los valores es menor a 8.8 eventos por año. Sin embargo, estos resultados no reflejan situaciones más o menos desfavorables, que tengan relación con la variabilidad de los índices. La simulación de Monte Carlo se presenta como una herramienta para obtener esta variabilidad.

\section{Resultados con método de Monte Carlo}

Las frecuencias acumuladas obtenidas por medio de simulación Monte Carlo se muestran en la figura 5, con resultados muy parecidos a los de la figura 4 .

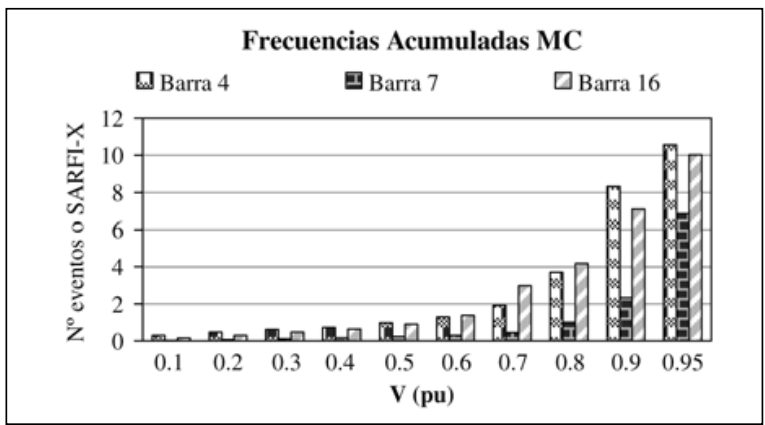

Figura 5. Frecuencias acumuladas MC en barras 4, 7 y 16 del sistema de prueba.

Para una mejor visualización, en la figura 6 se comparan los resultados obtenidos para los casos A, B y MC en la barra 16, donde se aprecia que los resultados de Monte Carlo se ajustan a los entregados en el caso B. El caso A muestra estimaciones más gruesas.

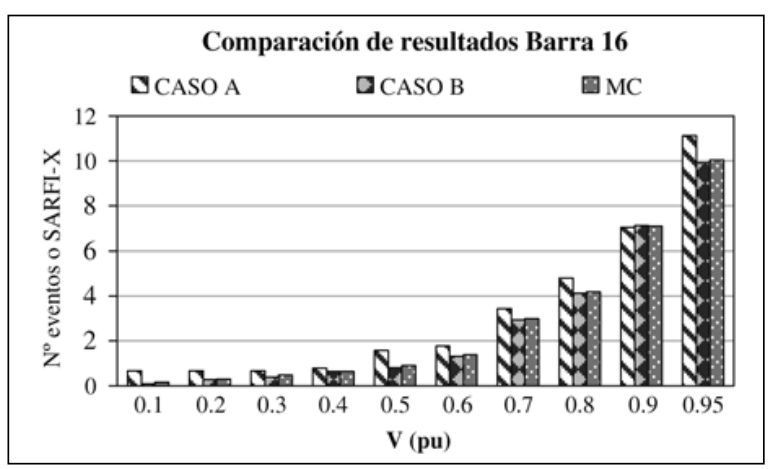

Figura 6. Comparación de resultados en barra 16, para los tres casos simulados.

La distribución de frecuencias entrega un espectro completo de posibles escenarios, que pueden constituir información importante para un cliente con cargas sensibles, conectadas en una barra en particular.

En la figura 7 se muestra el desempeño de la simulación y la media móvil en los primeros 37 años para SARFI-0.9 en la barra 4. Se observa que presenta un comportamiento irregular a lo largo del periodo de simulación; sin embargo, la media móvil converge al valor entregado en la figura 5 para el mismo SARFI-0.9, aproximadamente 8.35 eventos por año. 
Para el cálculo de la media móvil:

$$
\begin{aligned}
& M_{\text {edia_movil 1 }}=\frac{N^{\mathrm{o}} \text { dips }<0.9_{\text {año } 1}}{1} \\
& M_{\text {edia_movil 2 }}=\frac{N^{\mathrm{o}} \text { dips }<0.9_{\text {año } 1}+N^{\mathrm{o}} \text { dips }<0.9_{\text {año 2 }}}{2} \\
& M_{\text {edia_movil 3 }}=\frac{N^{\mathbf{o}} \text { dips }<0.9_{\text {año } 1}+N^{\mathbf{o}} \text { dips }<0.9_{\text {año } 2}+N^{\mathbf{o}} \text { dips }<0.9_{\text {año } 3}}{3} \\
& M_{\text {edia_movil. } n}=\frac{N^{\mathrm{o}} \operatorname{dips}<0.9_{a \tilde{n} o 1}+N^{\mathrm{o}} \operatorname{dips}<0.9_{a \tilde{n} O 2}+\ldots+N^{\mathrm{o}} \operatorname{dips}<0.9_{a \tilde{n} o n}}{n}
\end{aligned}
$$

Cuando $n$ tiende a infinito, el valor de la media móvil corresponde al entregado por el método de posiciones de falla.

El error de esta estimación puede ser medido por un intervalo de confianza como indicado en (20).

$$
\text { SARFI }-0.9 \in\left[\bar{X}-\frac{t_{\alpha / 2, n-1} \cdot s}{\sqrt{n}}, \bar{X}+\frac{t_{\alpha / 2, n-1} \cdot s}{\sqrt{n}}\right]
$$

Donde:

$\bar{X} \quad$ : Es el promedio de las muestras

s : Desviación estándar de la muestra

$\mathrm{t}_{\alpha / 2, \mathrm{n}-1}$ : Es el valor crítico correspondiente al $95 \%$ de confianza de la distribución t-student para n-1 grado de libertad.

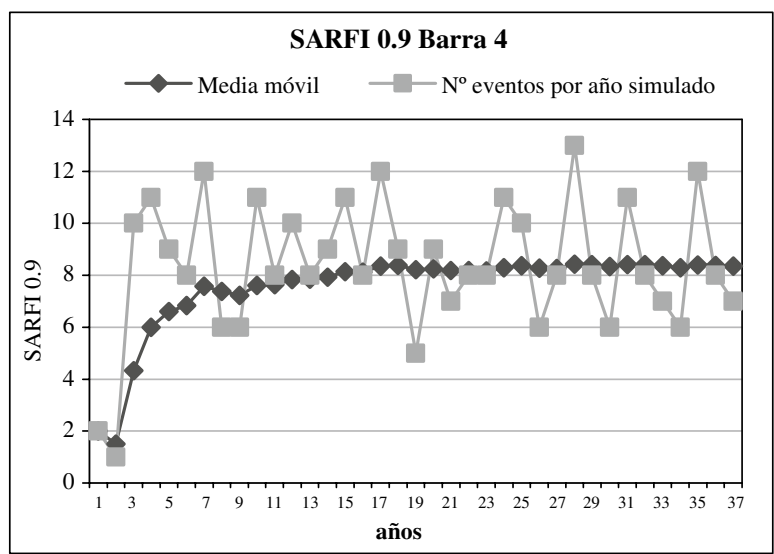

Figura 7. Media móvil y desempeño de la simulación para SARFI-0.9 en barra 4.

En la figura 7 se ilustra la media móvil para 37 años. Al considerar n-1 grados de libertad, es decir 36, resulta que $t_{\alpha / 2, n-1}=1.96$ (de una tabla t-student). Finalmente el intervalo de confianza del SARFI-0.9 es:

$$
\text { SARFI-0.9 } \in[7.34 ; 8.6]
$$

Esto quiere decir que, con un $95 \%$ de probabilidad, el SARFI-0.9 se encontrará entre 7.34 y 8.6 eventos por año.

En la figura 8 se muestran los resultados para la misma barra 4, pero para SARFI-0.7. Se observa que al ser los eventos de magnitud menor o igual a 0.7 del tipo severo, tienen menor ocurrencia. Así, en los años 5, 7, 21 y 32 no se presentan eventos de esta magnitud en la barra. El valor de largo plazo, representado por la media móvil sobre 37 muestras o años, es 1.91 .

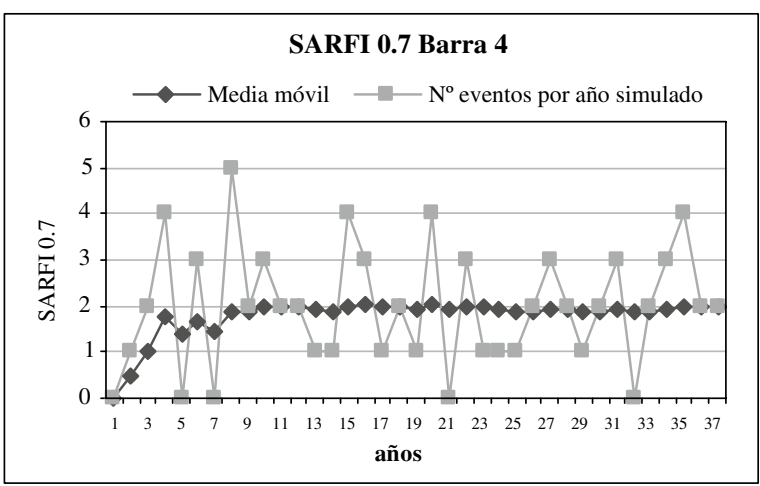

Figura 8. Media móvil y desempeño de la simulación para SARFI-0.7 en barra 4.

Para el SARFI-0.7 el intervalo de confianza aplicando (20) es:

$$
\text { SARFI-0.7 } \in[1.62 ; 2.37]
$$

En el caso de SARFI-0.7, el valor se encuentra entre 1.62 y 2.37 eventos por año, con un $95 \%$ de probabilidad. A partir de las curvas de desempeño de la simulación se pueden construir los gráficos de distribución de frecuencia. En las figuras 9 y 10 se muestra la distribución de frecuencia para SARFI-0.9 y SARFI-0.7 de la barra 4.

En la figura 9 se observa que, de los 1.000 años de simulación, aproximadamente en 170 se presentaron ocho eventos de magnitud menor o igual a $0.9 \mathrm{pu}$ en la barra 4 , por lo que el valor esperado para este índice tiende a 8.35; sin embargo, de esta figura también se concluye que en el $50 \%$ de los casos simulados (500 años) se presentaron más de ocho eventos de magnitud menor o igual a 0.9 pu, y que en el $18 \%$ de los casos se presentaron más de 10 eventos por año. Considerando que los dispositivos atenuadores de dips son de elevado costo, esta información puede ser un gran complemento para tomar alguna decisión. 


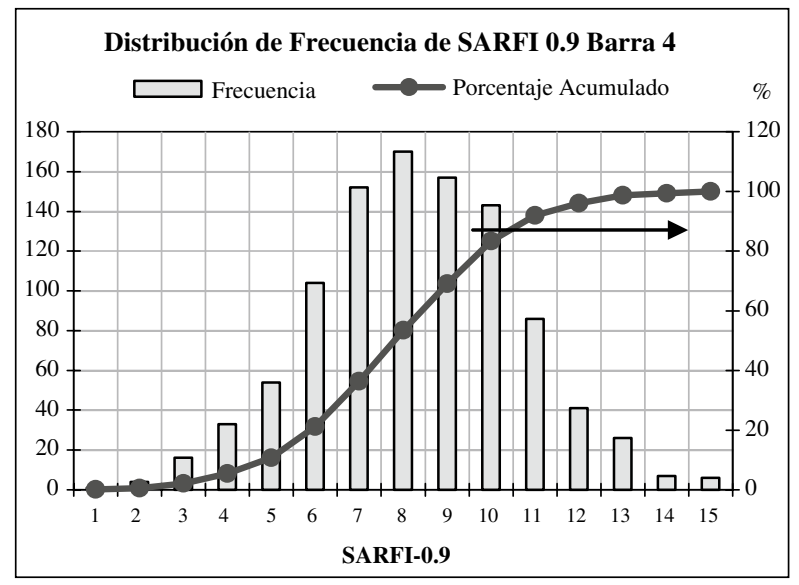

Figura 9. Distribución de frecuencias de SARFI-0.9 en barra 4.

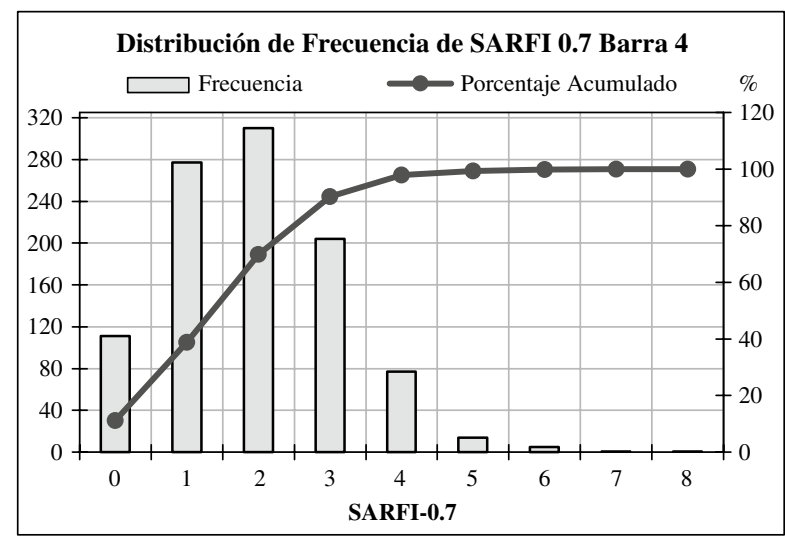

Figura 10. Distribución de frecuencias de SARFI-0.7 en barra 4.

En la figura 10 se muestra la distribución de frecuencias para SARFI-0.7 en la barra 4. Al ser eventos severos de menor ocurrencia, los valores se distribuyen poco hacia los lados. Además, se observa que en 115 años no se presentan eventos de esta magnitud; sin embargo, se pueden establecer conclusiones similares a las del caso anterior.

En la figura 11 se muestra la distribución de frecuencias para SARFI-0.7 en la barra 7. En esta barra se presentó solo un evento de magnitud menor o igual a 0,7 en 387 años, es decir el 38,7\% de los casos. Esta sería una buena barra para un cliente con procesos productivos sensibles a dips.

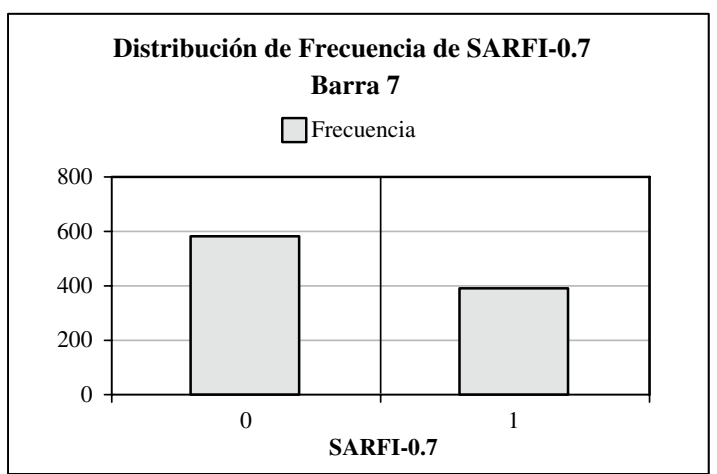

Figura 11. Distribución de frecuencias de SARFI-0.7 en barra 7 .

Otro aspecto interesante de analizar es el comportamiento de la desviación estándar de la distribución normal de los tiempos medios para falla (TMF). En la figura 12 se muestra la distribución de frecuencias para SARFI-0.9 en la barra 4, con una desviación estándar de $80 \%$ y un periodo de simulación de 3000 años. Se puede observar que al aumentar la desviación estándar de 30\% a $80 \%$ aparecen nuevas frecuencias. Así, aparecen años donde se presentan 16,17 y 18 eventos por año, que representan casos de baja ocurrencia. El intervalo de confianza para SARFI-0.9, pero con $\mathrm{DS}=80 \%$, es más grande que para la simulación con $\mathrm{DS}=30 \%$, debido a que los valores se distribuyen con una cantidad mayor de frecuencias.

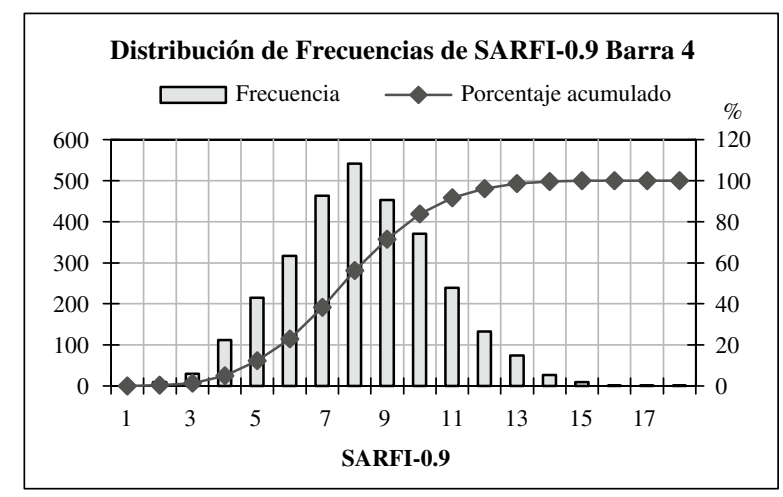

Figura 12. Distribución de frecuencias de SARFI-0.9 en barra 4 para $\mathrm{DS}=80 \%$ y 3.000 años de simulación.

SARFI-09 e [7.11; 8.87]

De esta forma, a partir de la estimación de valores de largo plazo, se pueden obtener valores promedios y percentiles95\%. En la tabla 3 se muestra esta estadística para una desviación estándar de $30 \%$. 
Tabla 3. Estadística obtenida vía simulación de Monte Carlo.

\begin{tabular}{|c|c|c|c|c|c|c|}
\cline { 2 - 7 } \multicolumn{1}{c|}{} & \multicolumn{6}{|c|}{ Estadísticas del Sistema por Magnitud (DS=30\%) } \\
\cline { 2 - 7 } & $\mathbf{0 . 1}$ & $\mathbf{0 . 3}$ & $\mathbf{0 . 5}$ & $\mathbf{0 . 7}$ & $\mathbf{0 . 9}$ & $\mathbf{0 . 9 5}$ \\
\hline Promedio & 0.186 & 0.468 & 0.907 & 1.974 & 6.818 & 9.845 \\
\hline Percentil-95 & 0.35 & 0.79 & 1.63 & 3.09 & 8.92 & 11.44 \\
\hline
\end{tabular}

Estos valores son similares a los entregados para el caso $\mathrm{C}$ de posiciones de falla, especialmente los de magnitud mayor a $0.7 \mathrm{pu}$; sin embargo, Monte Carlo entrega además la distribución completa para los índices del sistema, la cual se muestra en la figura 13, para los valores promedios. Se observa que el promedio que más se repite en el periodo de 1.000 años es 7.2 eventos de magnitud menor o igual a 0.9 pu. Además se observa que existen escenarios de peores condiciones, que, si bien son escasos, pueden producir efectos nocivos.

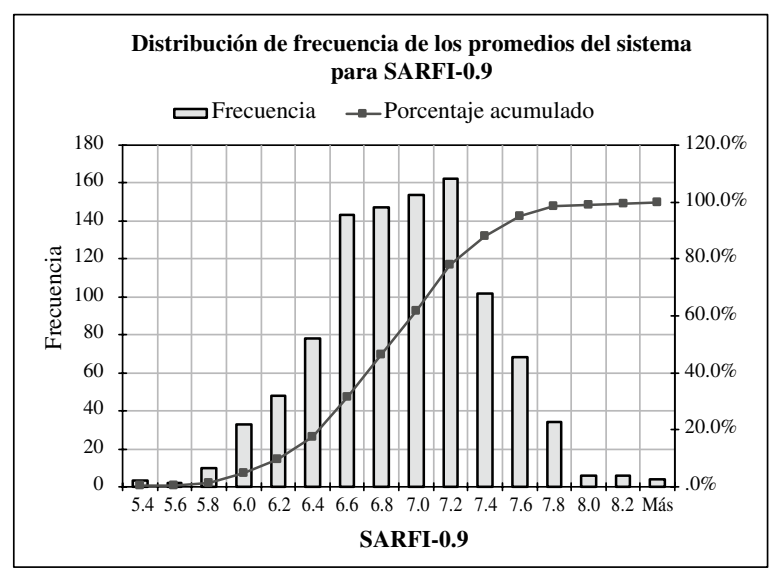

Figura 13. Distribución de frecuencias de los promedios del sistema, SARFI-0.9.

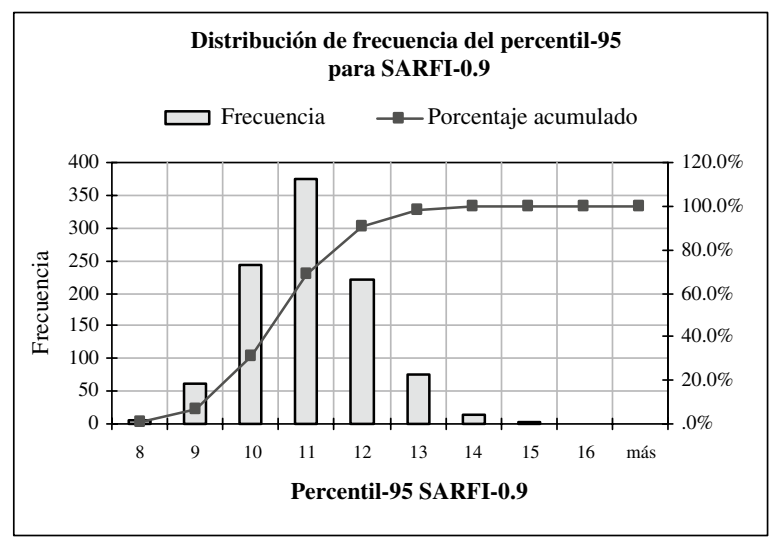

Figura 14. Distribución de frecuencias para percentiles-95 del sistema, SARFI-0.9.
En la figura 14 se muestra la distribución del percentil-95 del sistema, de donde se pueden obtener las mismas conclusiones que en el caso de la distribución de los promedios.

\section{CONCLUSIONES}

La estimación estocástica de dips es una herramienta importante de predicción, en la medida que los sistemas de potencia no cuentan con esquemas completos y complejos de monitoreo de calidad de energía. Los resultados de una estimación de dips son útiles para ser comparados con la compatibilidad de equipos sensibles a este tipo de perturbaciones.

Para realizar estudios específicos se puede emplear el método de posiciones de falla, que es una herramienta poderosa para la estimación estocástica de dips de largo plazo. Sin embargo, la precisión de sus resultados depende de las posiciones de falla consideradas (cantidad y tipo), entre las que eventualmente podrían quedar fuera algunas situaciones de mayor impacto.

Para evitar esta situación, en el trabajo se ha utilizado la simulación de Monte Carlo, obteniendo una distribución completa de frecuencias y la variabilidad de índices. Esto es importante debido a que, generalmente, la operación de una planta o industria se proyecta para horizonte superior a 30 años, periodo durante el cual es muy difícil precisar la ocurrencia de dips.

La simulación de Monte Carlo es sensible a los modelos que describen las variables aleatorias, por lo que todos los resultados entregados en este trabajo pueden ser mejorados. Como ejemplo, se mostró el efecto de cambiar la desviación estándar para los tiempos medios de falla. Algo similar es aplicable a la modelación del sistema, la que puede ser mejorada incorporando, entre otros aspectos, la impedancia de falla y una representación detallada del comportamiento de las cargas.

\section{REFERENCIAS}

[1] M.H.J. Bollen. "Understanding Power Quality Problems: Voltage Sags and Interruptions". IEEE Press Series on Power Engineering. New York. 2000.

[2] V.E. Wagner, A.A. Andreshak and J.P. Staniak. "Power quality and Factory Automation". IEEE Transactions on Industry Applications Vol. 26 $\mathrm{N}^{\circ}$ 4, pp. 620-626. 1990. 
[3] M. McGranaghan. "Effects of Voltage Sags in Process Industry Applications". Proceedings of Stockholm Power Tech International Symposium on Electric Power Engineering, Stockholm. Sweden. June 1995.

[4] R.C. Leborgne, G. Olguin and M.H.J. Bollen. "Sensitivity Analysis of Stochastic Assessment of Voltage Dips". IEEE Power Conference. Singapore. November 2004.

[5] Curve Application Note. Information Technology Industry Council (ITIC). Washington, USA. URLs: http://www.itic.org/technical/iticurv.pdf

[6] A. Leon-Garcia. "Probability and Random Processes for Electrical Engineering". Addison-Wesley Publishing Company. Inc. 1994.

[7] G. Olguin and M.H.J. Bollen. "Stochastic Assessment of Unbalanced Dips in Large Transmission Systems". IEEE Power Tech Conference Proceedings 2003. Vol. 4, pp. 8. Bologna, Italy. June 23-26. 2003.

[8] “The IEEE Reliability Test System-1996”. Power Systems. IEEE Transactions. Vol. $14 \mathrm{~N}^{\circ} 3$, pp. 10101020. Agosto 1999.

[9] H. Brown. "Grandes Sistemas Elétricos - Métodos Matriciais". Livros Técnicos e Científicos Editora S.A. Rio de Janeiro. 1977.
[10] G. Olguin. "Stochastic Assessment of Voltage Dips Caused by Faults in Large Transmission System". Tesis para al grado de Licentiate of Engineering, Chalmers University of Technology. 2003.

[11] ANSI C57.21.10. "American National Standard for Transformers 230kV and below 833/958 through $8333 / 10417 \mathrm{kVA}$, single-phase, and 750/862 through 60000/80000/100000 kVA, three-phase without load tap changing; and 3750/4687 through 60000/80000/100000 kVA with load tap changingSafety requirements". 1988.

[12] Y.S. Lim and G. Strbac. "Analytical approach to probabilistic prediction of voltage sags on transmission networks". Generation, Transmission and Distribution. IEE Proceedings. Vol. $149 \mathrm{~N}^{\circ} 1$, pp. 7-14. Enero 2002.

[13] G. Olguin and M. H. J. Bollen. "The method of fault positions for stochastic prediction of Voltage Sags: A case Study". Proceeding of $7^{\text {th }}$ International Conference of Probabilistic Methods Applied to Power Systems. Vol. 2, pp. 557-562. Nápoles, Italia. Septiembre 22-26. 2002. 